\title{
BMJ Open A randomised double-blind control study of early intracoronary autologous bone marrow cell infusion in acute myocardial infarction (REGENERATE- AMI)
}

\author{
Stephen Hamshere, ${ }^{1,2}$ Tawfiq Choudhury, ${ }^{1,2}$ Daniel A Jones, ${ }^{1,2}$ Didier Locca, ${ }^{1}$ \\ Peter Mills, ${ }^{1}$ Martin Rothman, ${ }^{1}$ Charles Knight, ${ }^{1}$ Mahesh Parmar, ${ }^{5}$ Samir Agrawal, ${ }^{3}$ \\ John Martin, ${ }^{4}$ Anthony Mathur ${ }^{1,2}$
}

To cite: Hamshere $S$, Choudhury $\mathrm{T}$, Jones DA, et al. A randomised doubleblind control study of early intracoronary autologous bone marrow cell infusion in acute myocardial infarction (REGENERATE-AMI). BMJ Open 2014;4:e004258. doi:10.1136/bmjopen-2013004258

- Prepublication history and additional material for this paper is available online. To view these files please visit the journal online (http://dx.doi.org/10.1136/ bmjopen-2013-004258).

Received 16 October 2013 Revised 30 December 2013 Accepted 14 January 2014

CrossMark

For numbered affiliations see end of article.

Correspondence to Professor Anthony Mathur; a.mathur@qmul.ac.uk

\section{ABSTRACT}

Introduction: Acute myocardial infarction (AMI) remains a major cause of mortality and morbidity worldwide despite the latest therapeutic advances designed to decrease myocardial injury. Preclinical and emerging clinical evidence show that the intracoronary injection of autologous bone marrow mononuclear cells (BMCs) following AMI leads to improvement in left ventricular ejection function (LVEF). In this clinical trial we will for the first time assess the effect of early $(<24 \mathrm{~h})$ infusion of autologous BMCs following AMI on cardiac function.

Methods and analysis: REGENERATE-AMI is a double-blind, randomised, multicentre, placebocontrolled trial to determine whether early $(<24 \mathrm{~h})$ intracoronary infusion of BMCs improves LVEF after AMI. The study will enrol 100 patients presenting with an anterior AMI demonstrating anterior regional wall motion abnormality. Patients will be randomised to receive intracoronary infusion of BMCs or placebo $(0.9 \%$ saline). Primary endpoint will be change in LVEF at 1 year compared to baseline, measured by cardiac MRI. Secondary endpoints at 6 months include the change in global LVEF relative to baseline measured by quantitative left ventriculography and echocardiography, as well as major adverse cardiac events which is also measured at 1 year.

Ethics and dissemination: The study will be performed in agreement with the Declaration of Helsinki and is approved by local ethics committee (NRES Committee London West London: 07/Q0603/76).

Trial registration: $h t t p: / / c l i n c i a l t r i a l s . g o v$ (NCT00765453). The results of the trial will be published according to the CONSORT statement and will be presented at conferences and reported in peer-reviewed journals.

\section{INTRODUCTION}

Cardiovascular disease (CVD) remains the most common cause of death in the world,

\section{Strengths and limitations of this study}

- This is the first randomised control trial testing early infusion of bone marrow mononuclear cells to improve left ventricular ejection function.

- REGENERATE-AMI is a multicenter clinical trial of cell therapy.

- Each centre will use local cell processing facilities following a standardised protocol.

accounting for an estimated 17.3 million deaths in 2008, representing $30 \%$ of all deaths worldwide. ${ }^{1}$ In the UK CVD accounts for almost 191000 deaths each year, almost half $(46 \%)$ of which is from ischaemic heart disease (IHD). IHD is the most common cause of death in the UK (mainly as a consequence of acute myocardial infarction (AMI)), causing approximately 82000 deaths per year, and accounting for £29.1 billion pounds1 within the National Health Service (NHS). ${ }^{1}$

Primary angioplasty has significantly improved the outcome for patients with AMI, however there remains a significant risk of mortality at 30 days $(7 \%)$ despite optimal medical management. ${ }^{2}$ As treatment improves, there is an increase in the numbers of survivors of AMI but this means that there is also an associated increase in the incidence of heart failure (15$20 \%$ ) in patients who still develop significant myocardial damage. ${ }^{3}$ While early reperfusion therapy directly decreases the amount of myocardial necrosis, a consequence of ischaemic damage, at least $50 \%$ of the final infarct size results from the damage caused by ischaemia-reperfusion (I-R) injury. ${ }^{4}{ }^{5}$ This means that there are two targets for therapeutic intervention in the early stages of AMI; decreasing initial myocardial ischaemia by 
prompt restoration of coronary flow and preventing the damage caused by I-R injury as the blood supply is restored. While early reperfusion is achieved by primary percutaneous coronary intervention (PPCI) it is possible that cell therapy may play a role in limiting the I-R injury. ${ }^{6}$ This, therefore, provides an additional rationale for administering cell therapy at an early stage after myocardial infarction, which is independent of the concept of cardiac regeneration and has important therapeutic potential.

\section{THE HEART AND REGENERATION}

Until recently the heart has been considered as a terminally differentiated organ with no ability to self repair (cf hepatic regeneration). ${ }^{7}$ However, recent studies ${ }^{8-10}$ imply that there may be a background level of cellular renewal by engraftment and differentiation of primitive cells. Limited regeneration could occur through circulating primitive bone marrow mononuclear cells (BMCs) that could engraft in the heart in response to injury ${ }^{11}$ or from a resident cardiac stem cell population. ${ }^{12}$ Clearly, this reparative potential is not capable of an adequate response to significant myocardial damage but the discovery of this endogenous repair process has provided a further rationale for the use of cell therapy based on the amplification of this natural process.

\section{STEM CELL THERAPY AND MYOCARDIAL INFARCTION}

The seminal work by Orlic et $a l^{13}$ in 2001 demonstrated a significant increase in cardiac function following injection of allogeneic bone marrow-derived cells $3-5 \mathrm{~h}$ after a mechanically induced myocardial infarction in a mouse model. In further separate preclinical studies in which progenitor cells were injected $1 \mathrm{~h}$ after induction of myocardial infarction an angiogenesis-type response was seen in the release of angiogenic ligands and related cytokines compared to the controls. ${ }^{14-16}$ Recent experiments using mouse models have shown that earlier infusion time (immediate, compared to 1 week) and higher cell concentration has a greater impact on left ventricular (LV) function post-mechanical infarction. ${ }^{17}$ These studies, therefore, support the rationale of the trial design reported here for attempting to deliver cell therapy as soon as possible after AMI.

\section{AMI STEM CELL THERAPY IN MAN}

To date, the majority of clinical trials evaluating stem cell therapy after AMI have been phase I or II studies designed to look for safety and feasibility with surrogate measures for early signs of efficacy. The first randomised trial investigating cell use post-AMI was TOPCARE-AMI. This showed an improvement in the LV function from $51.6 \pm 9.6 \%$ at baseline to $60.1 \pm 8.6 \%$ at 4 months in the cell therapy arm vs $51.0 \pm 10 \%$ at baseline to $53.5 \pm 7.9 \%$ in the control arm. ${ }^{18}$ Improvement in patients treated with cell therapy was also seen in the BOOST study (bone marrow transfer to enhance ST elevation infarction regeneration) that demonstrated a $6.7 \%$ improvement in $\mathrm{LV}$ function at 6 months from baseline in the stem cell arm compared to $0.7 \%$ in the control arm $(\mathrm{p}=0.0026) .{ }^{19}$ The largest randomised control trial to date is the REPAIR-AMI trial (intracoronary progenitor cells in AMI). This double-blinded, placebo-controlled trial of 204 patients assessed LV function change after 4 months in patients treated with autologous bone marrow-derived cell therapy compared to controls. REPAIR-AMI showed a statistically significant improvement in LV function in cell-treated patients compared to the control arm $(5.5 \pm 7.3 \%$. vs $3.0 \pm 6.5 \%, \mathrm{p}=0.01) .^{20}$ Further follow-up of patients with REPAIR-AMI has demonstrated a reduction in mortality in the cell-treated group although the trial was not statistically powered to assess this.

In contrast to these studies, a number of clinical trials have been published that failed to reproduce these positive findings. In the LEUVEN-AMI, Janssens et al, reported no change in global left ventricular ejection function (LVEF) after autologous BMCs administration $24 \mathrm{~h}$ following myocardial infarction. Subgroup analysis for this trial did however demonstrate a reduction in infarct size in cell-treated patients compared to controls. It is not clear why this improvement did not lead to beneficial effects on $\mathrm{LV}$ function. ${ }^{21}$ In addition, ASTAMI $^{22}$ and HEBE $^{23}$ trials have both failed to show beneficial effect of BMCs on LVEF and LV volumes in patients with AMI. Recently published trials have examined the effect of the timing of infusion of cell therapy on outcome. The LATE TIME study, a double-blinded, randomised, controlled trial of 87 patients showed no improvement in LVEF after cell infusion therapy at 2-3 weeks post-AMI. ${ }^{24}$ The TIME trial (a randomised controlled trial (RCT) of 120 patients) also failed to show improvement in LVEF with a late infusion at 7 days postinfarction. Interestingly, this trial also looked at infusion at 3 days postinfarction but unlike REPAIR-AMI and BOOST it failed to show statistically significant improvement in LV function. ${ }^{25}$ In addition to this, the literature has not reported any significant harm following early injection of BMCs in animal models, despite the views of some that this may be hazardous either to the subject or the cell products. The aim of REGENERATE-AMI is to ensure that this lack of evidence is addressed in the safety analysis of the trial.

There has been considerable debate regarding the discrepancies in the results of these clinical trials and also the failure to translate the success of preclinical experiments into man. ${ }^{13}$ Possible explanations include variations in the cell processing methods, the use of adjunctive therapies and the patient population, highlighting the need for a standardised, widely applicable approach in order to assess whether cell therapy has a role in the management of myocardial infarction.

Bearing in mind these issues and the preclinical benefits of early delivery of cell therapy, REGENERATE-AMI has been designed to establish for the first time whether 
an early time point for delivery of cells $(<24 \mathrm{~h})$ is safe, beneficial and feasible when used as an adjunct to the established treatment of AMI. Patients presenting to heart attack centres with evidence of large infarcts (anterior territory) will have their bone marrow taken and then be randomised to receive either the mononuclear fraction from this sample or saline alone. This phase II clinical trial is novel in the timing of the infusion of the bone marrow-derived product (less than $24 \mathrm{~h}$ following index revascularisation procedure). This time point will be the closest to that used in the preclinical models, which demonstrated a significant benefit in restoration of cardiac function. Additionally, the inclusion of only anterior AMIs will target the patient population most likely to benefit as identified in the earlier trials. ${ }^{13-17}$ The cell preparation technique will use the methodology of the REPAIR-AMI trial.

\section{METHODS AND ANALYSIS \\ Study design}

REGENERATE-AMI is a double-blind, randomised, multicentre, placebo-controlled trial to determine whether the intracoronary infusion of autologous bone marrow progenitor cells is feasible, safe and shows positive evidence of activity (ie, improves cardiac function) after an acute anterior myocardial infarction (see figure 1) when administered in addition to standard care.

\section{Aims}

1. To demonstrate that it is safe and feasible to deliver autologous bone marrow-derived stem cells within $24 \mathrm{~h}$ of the primary angioplasty procedure (as opposed to days as in previous studies).

2. To demonstrate that administration of autologous bone marrow-derived stem cells (in addition to standard care) leads to improvement in cardiac function as measured by improvement in ejection fraction as measured by cardiac advanced imaging (cardiac MRI (CMR) or CT).

\section{Efficacy hypothesis}

Intracoronary infusion of autologous bone marrowderived progenitor cells in patients who have undergone successful PPCI for acute anterior myocardial infarction will lead to an improvement in cardiac function greater than that seen by placebo alone.

\section{Study participants}

This multicentre trial will recruit 100 patients who have been admitted with a diagnosis of acute AMI treated successfully with PPCI. Only patients with an AMI as demonstrated by an anterior wall motion abnormality on left ventriculography and with a culprit lesion in the left anterior descending coronary artery will be eligible for the study (see boxes 1 and 2). Patients with lesions in other vessels that require treatment will be included as long as they are treated during the index procedure

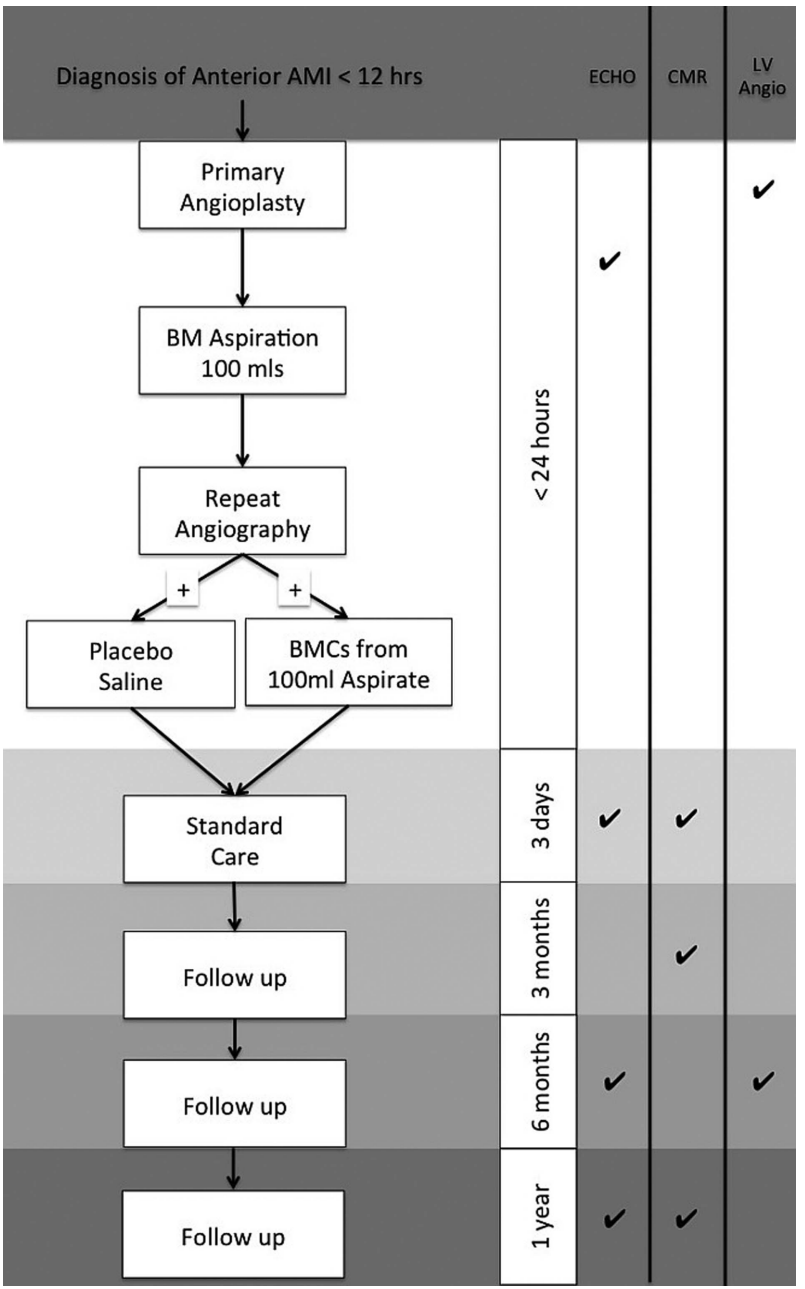

Figure 1 Flow diagram of study design. AMl, acute myocardial infarction; BM, bone marrow; CMR, cardiac magnetic resonance imaging; ECHO, echocardiogram; LV, left ventricular.

with the culprit vessel. Patients requiring staged procedures will be excluded. Ptential patients will be approached following the index primary PCI procedure.

\section{Box 1 Inclusion criteria}

Patients have to fulfil the following criteria:

- Patients presenting with acute anterior myocardial infarction (ST elevation in at least 2 contiguous anterior leads $\geq 0.2 \mathrm{mV}$ ) and treated with acute percutaneous coronary intervention (PCI) with stent implantation within $24 \mathrm{~h}$ after symptom onset.

- Acute $\mathrm{PCl} /$ stent implantation has been successful (residual stenosis visually $<30 \%$ and TIMI flow $\geq 2$ ).

- At the time of inclusion the patient no longer requires intravenous catecholamines or mechanical hemodynamic support (aortic balloon pump).

- Significant regional wall motion abnormality on left ventricular angiogram in left anterior descending (LAD) territory at the time of acute $\mathrm{PCl}$.

- Age 18-80 years (primary angioplasty confers an adverse prognosis in those over the age of 80 years).

Written informed consent. 


\section{Box 2 Exclusion criteria}

Patients are excluded if one of the following criteria is met:

- Regional wall motion abnormality not consistent with culprit vessel;

- Need to revascularise additional vessels, outside the infarct artery as a planned procedure (these vessels can be treated at baseline);

- Arteriovenous malformations or aneurysms;

- Active infection, or fever or diarrhoea within the past 4 weeks;

Chronic inflammatory disease;

Known HIV infection or active hepatitis;

- Neoplastic disease without documented remission within the past 5 years;

- Cerebrovascular insult within 3 months;

- Impaired renal function (creatinine $>200 \mathrm{mmol}$ ) at the time of cell therapy;

- Significant liver disease (gamma-glutamyltrasferase $>2 \times$ upper limit) or spontaneous International Normalised Ratio $>1.5$ );

- Anemia (haemoglobin $<8.5 \mathrm{mg} / \mathrm{dL}$ );

- Platelet count <100 000/ $\mu \mathrm{L}$;

Hypersplenism;

- Known allergy or intolerance to clopidogrel, heparin or abciximab;

- History of bleeding disorder;

- Gastrointestinal bleeding within 3 months;

- Major surgical procedure or trauma within 2 months;

- Uncontrolled hypertension;

- Pregnancy;

- Mental retardation leading to inability to obtain informed consent;

Previously performed stem/progenitor cell therapy;

Participation in another clinical trial within the past 30 days.

Centres participating in the trial are The London Chest Hospital (London, UK), Royal Free Hospital (London, UK), Heart Hospital (London, UK), Centre Hospitalier Universitaire Vaudois (Lausanne, Switzerland) and Rigshospitalet (Copenhagen, Denmark).

\section{Randomisation and study blinding}

Patients will be randomised to receive autologous BMCs or matching placebo (in a 1:1 block randomisation allocation) with the patient and treating clinicians blinded to the assignment (double-blind). The randomisation process will be performed by dedicated trial software IHD CLINICAL. This is a web-based password secured and encrypted data management system designed specifically for clinical trials in this area.

\section{Bone marrow aspiration and processing}

Following consent, patients will undergo bone marrow aspiration performed by a trained member of the research team. The patient will be rolled onto their side and local anaesthetic administered into the posterior superior iliac spine. One hundred millilitres of bone marrow will be aspirated into heparinised syringes and the contents transferred to a specimen bag for transport to the stem cell unit. The bone marrow is passed to the cell culture laboratory of the hospital where the bone marrow aspiration has been performed. The progenitor cells will be isolated using Ficoll-Hypaque centrifugation. The protocol for stem cell preparation has been previously set out by Schächinger et $a l^{26}$ The autologous BMCs or placebo will be placed in identical sterile syringes and transferred back to the cardiac catheter laboratories for the infusion procedure (Heparin will not be included in the final solution for reinfusion).

\section{Infusion procedure}

Patients will return to the cardiac catheterisation laboratory once the cell processing is complete and the cells are ready for collection (ideally within $6-8 \mathrm{~h}$ ). A bolus of body-weight-adjusted heparin will be given if the ACT is $<200$. Progenitor cell infusion will be performed in accordance with the protocol developed by Professor Zeiher and collegues. ${ }^{20}$ A guide catheter will be used to intubate the left coronary ostium and a 0.014-inch guidewire will be introduced in the left anterior descending artery. A $10 \mathrm{~mm}$ long 'over-the-wire' balloon catheter will be positioned within the segment of the previously implanted stent. The balloon diameter should be $\pm 0.5 \mathrm{~mm}$ the size of the implanted stent, to achieve a complete vessel occlusion in the stented segment during low-pressure balloon inflation. The balloon catheter, used for the infusion of the cells, will be occluded with low pressure $(<2-4$ bar $)$. Prior to each cell infusion, complete occlusion of the vessel will be documented angiographically by injection of some contrast agent in the vessel. Ten millilitres of the cell suspension will be infused in three portions (each $3.3 \mathrm{~mL}$ ) during $3 \mathrm{~min}$ occlusion time for each portion. After application of the first portion of the cell suspension, the balloon will be deflated for $3 \mathrm{~min}$, prior to the next occlusion in order to avoid extensive ischaemia. Prior to the first balloon occlusion and finally, after completion of the cell infusion procedure, angiographically estimated coronary blood flow and the integrity of the treated will be documented and assessed by coronary angiography. This method has been used in previous trials with the aim to allow for adhesion and potential transmigration of the infused cells through the endothelium. $^{18}$

\section{Study endpoints}

Blood samples are taken for local measurement of FBC, U\&E, LFTs, TFTS, creatine kinase, cardiac troponin T, C reactive protein and brain natriuretic peptide. These are again measured at the 6-month and 12-month visits. Blood samples are also taken for HIV, hepatitis B and C, HTLV and syphilis at time of randomisation, this is required by the Stem Cell Laboratory for quality assurance. Plasma samples will be obtained for storage in cryovials at $-80^{\circ} \mathrm{C}$ in $\mathrm{UK}$ centres only. These samples will be used for further analysis and can be stored for up to 10 years in marked cryovials and in a locked $-80^{\circ} \mathrm{C}$ freezer with thaw protection system installed. 


\section{Primary endpoint}

The primary efficacy endpoint for the study will be the change in LVEF between baseline and 12 months assessed by CMR. In patients who are clinically unable to undergo CMR a cardiac CT scan will be performed as an alternative.

Secondary endpoints include infarct size and myocardial salvage at 3 months (CMR), change in global ejection fraction as measured by $\mathrm{LV}$ angiography after 6 months (quantitative analysis), and improvement in LV function at 6 and 12 months as assessed by echocardiography. Clinical endpoints include the acute safety and tolerability of intracoronary stem cells following ST elevation myocardial infarction (haemodyamics and inpatient major adverse cardiac events (MACE), heart failure status (NYHA), heart failure admissions, quality of life (UK sites only) and assessment of MACE endpoints at 6 and 12 months (death (independent of cause and sudden death), myocardial infarction (Q-wave and non-Q-wave), coronary revascularisation \{(coronary bypass or PCI)\}). Further safety endpoints such as rates of cardiac device, that is, implantable cardioverter defibrillator (ICD) insertion will be recorded at 1 year.

All data will be anonymised prior to analysis, which will be performed by an independent member of the department not associated with the trial. Scans will be stored on a separate storage device to the reporting system-either CD or encrypted hard drive.

\section{Cardiac MRI}

Each patient will undergo CMR on the day of hospital discharge (Day 3), 3 months and 1 year following PPCI using each site's local CMR scanner. Each examination will use cine-CMR for ventricular volumes and function, and delayed enhancement (DE)-CMR for infarct size assessment and evaluation of microvascular obstruction. Myocardial oedema will be assessed at all time points using three slice T2-weighted triple inversion turbo spin echo short TI inversion recovery imaging. Myocardial salvage will be calculated from the T2 imaging and infarct size on the Day 3 scan. The inversion time will be optimised to null normal myocardium. Images will be analysed in a blinded fashion by two experienced operators. Scar and oedema volumes will be calculated by manually drawing endocardial and epicardial contours followed by semiautomated selection of normal remote myocardium per slice. Infarct size will be calculated using the full-width half maximum method as previously described. ${ }^{27}$

\section{CT imaging}

Patients who are unable to undergo CMR will be imaged using cardiac CT (approximately 5\% of patients). Analysis will use specialised cardiac CT circulation software with intraventricular tracings of end systolic and end diastolic timings.

\section{Quantitative left ventriculography}

The angiographic endpoint of the study is the change in global LVEF at 6 months relative to baseline measured by quantitative left ventriculography. LV angiography is used as a secondary endpoint for the purpose of comparison to early trials such as REPAIR-AMI, ${ }^{20}$ that also used LV angiographyas an endpoint. Left ventricular angiography is performed in the $30^{\circ} \mathrm{RAO}$ position and recorded at a minimum of 15-30 frames per second. Contrast is injected over $10 \mathrm{~s}$. At least two well-opacified sinus beats that are not postpremature beats must be obtained. A simultaneously recorded ECG must be supplied as well as the aortic and LV pressures before and after contrast injection. LVEF and volumes are calculated with the centreline method.

\section{Echocardiography}

Echocardiography will be used to assess LV systolic function using wall motion scoring and calculation of ejection fraction using Simpson's rule. Echocardiography will be performed using imaging protocols that will be standardised to ensure conformity. Assessment of LV function will be performed on the day of PPCI prior to stem cell infusion, 3 days after the PPCI (prior to discharge), at 6 months and also at 1 year to track the changes in cardiac function that occur over time as part of this study.

\section{Adjudication of potential endpoints}

All measurements will be assessed and adjudicated by a blinded expert. In the event of poor recordings, the expert decides whether the measurement at hand is of sufficient quality to be taken into the endpoint evaluation. Measurements of insufficient quality are dealt with in the analysis in the same manner as missing measurements. Potential MACE events will be assessed by a blinded Safety Committee. All core laboratory readings and adjudications will be completed and entered into the computer database before the study is unblinded.

\section{Statistical analysis}

To calculate the target sample size for the trial, an absolute increase in ejection fraction of $6 \%$ compared to baseline as measured by CMR will be considered significant and consistent with previous studies that used advanced imaging to measure endpoints. ${ }^{19}$ The published SD for these observations is approximately $8 \%$ and in keeping with what has been observed by Janssens $e t a l^{21}$ For a statistical power of $90 \%$ and a probability of a type I error of 0.05 using a two-sided t test, with an estimated $25 \%$ dropout at 1 year we will recruit a total of 100 patients in this study. Previous trials have shown a $2 \%$ improvement in patients who underwent PPCI alone. ${ }^{28}$ Therefore, using this information we will perform a post hoc analysis to compare the two groups, which will have $80 \%$ power to detect a $4 \%$ difference.

The analysis of the data will be based on the intention-to-treat principle. Baseline demographic and 
clinical variables are summarised for each randomised arm of the study. Continuous variables will be presented as mean (SD) or median (IQR). Categorical data are presented by frequencies and percentages.

The statistical comparisons within the treatment arms with respect to the primary endpoint will be performed using the independent-samples $t$ test as the principal analytic tool. Further statistical comparison between treatment arms with respect to the primary endpoint will be performed using the analysis of covariance including LVEF values at 12 months as dependent variables and the associated baseline values and the factor treatment as independent variables. Estimates of the treatment effect are presented together with $95 \%$ CI. Comparisons will be between the stem cell-treated and placebo control-treated group for the primary and secondary outcomes. Estimates of the treatment effects will be presented together with $95 \%$ CIs. All p values will be two-sided. ${ }^{29}$

For clinical outcomes such as the incidence of MACE (predefined clinical scenarios; death, myocardial infarction, re-admission for heart failure or revascularisation), Kaplan-Meier curves displaying the pattern of events during the 6-month and 12-month follow-up period are drawn. Statistical significance and 95\% CIs are calculated using Cox's proportional hazards model.

\section{Adverse events reporting}

An independent Data and Safety and Monitoring Board (DSMB) will be formed to monitor patient safety as the study progresses. The DSMB has been selected by and communicates directly to the study's Executive Committee. The DSMB will meet prior to initiation of the clinical study to determine whether stopping rules are needed or appropriate. If a serious concern with the safety of the patients in the trial would arise, the DSMB may recommend early termination of the study.

The DSMB will review safety issues throughout the trial which will include unexpected serious adverse events, mortality, complications of bone marrow aspiration, complications of the intracoronary infusion, complications of (diagnostic) study procedures and serious or life-threatening arrhythmia's. Every 3 months, all safety events are reported to the DSMB statistician, who will prepare tabulations by treatment groups. Fatalities are reported on a case-by-case basis.

\section{Ethical considerations}

Important ethical issues to consider with this type of trial are the need to obtain informed consent in the acute setting, the possible risk of BMCs use, the requirement for further interventional angiography and infusion shortly after the index procedure.

There are challenges in obtaining informed consent from an acutely unwell patient with AMI, as both the patient's physical condition and treatment with pain relief have the potential to make this difficult. A number of steps will be taken to deal with this. First is to ensure that patients are in a stable clinical condition when they come back for repeat procedures, therefore we have excluded patients who are unconscious, critically unstable (cardiogenic shock) and deemed unable to consent (ie, unable to understand in local language). Second, all patients will be provided with a patient information sheet prior to consent and the study will be discussed again with the patient after the procedure when they are on the ward. Lastly, if the patient has been given pain relief treatment or sedation during the initial PPCI that could alter their decision-making we shall ensure that the patient has enough time to recover prior to consent.

BMCs are the patients' own cells, therefore there is no risk of an allergic reaction. The repeat angiography and the infusion of BMCs or placebo into the coronary artery could lead to possible complications but this will be reduced by:

A. Using a low pressure inflation of the coronary balloon that is $0.5 \mathrm{~mm}$ smaller than the stented segment to reduce any dissection.

B. The infusion time will be divided into 3 min timed slots with 3 min to reduce further ischaemia.

\section{Dissemination}

The study will be performed in agreement to the Declaration of Helsinki. Data collection will be completed by mid-2014. Primary and secondary analysis will start immediately after data monitoring is completed. Publications will be prepared for submission in late 2014 . The results of the trial will be published according to the CONSORT statement. Dissemination of results will focus on publications in peer-reviewed journals, presentations at both national and international cardiology meetings and National Health Service (NHS) groups. In accordance with recommendations, REGENERATE-AMI has been registered with a public registry ClinicalTrials.gov.

\section{Summary}

REGENERATE-AMI will be the first RCT of autologous bone marrow-derived cells delivered to patients less than $24 \mathrm{~h}$ after presentation with acute AMI. The results of the study will demonstrate whether such early delivery is achievable and safe. The efficacy results of this trial will complete a time course for cell administration that has been complied as a result of previous studies and will therefore provide valuable information for the planning of future phase III clinical trials using autologous cell therapy in AMI.

\section{Author affiliations}

${ }^{1}$ Department of Cardiology, London Chest Hospital, Barts Health NHS Trust, London, UK

${ }^{2}$ Queen Mary University of London, Cardiology, London Chest Hospital, NIHR Cardiovascular BRU, London, UK

${ }^{3}$ Stem Cell Laboratory, Barts Health NHS Trust and Blizard Institute, Queen Mary University of London, London, UK

${ }^{4}$ British Heart Foundation Laboratories, Department of Medicine, University College London, London, UK

${ }^{5}$ MRC Clinical Trials Unit, London, UK 
Contributors TC, SH, DAJ, CK, AM and MP participated in preparation and writing of manuscript. SA, CK, DL, AM, JM, PM, MP and MR participated in trial design. MP participated in statistics.

Funding This work was supported by grant from the UK Stem Cell Foundation, Heart Cells Foundation and Barts and the London Charity.

Competing interests None.

Ethics approval NRES Committee London West London: 07/Q0603/76.

Provenance and peer review Not commissioned; externally peer reviewed.

Open Access This is an Open Access article distributed in accordance with the Creative Commons Attribution Non Commercial (CC BY-NC 3.0) license, which permits others to distribute, remix, adapt, build upon this work noncommercially, and license their derivative works on different terms, provided the original work is properly cited and the use is non-commercial. See: http:// creativecommons.org/licenses/by-nc/3.0/

\section{REFERENCES}

1. WHO. Global atlas on cardiovascular disease prevention and control. WHO, W.H. Federation, and W.S. Organization, 2011:164

2. Widimsky P, Wijns W, Fajadet J, et al. Reperfusion therapy for ST elevation acute myocardial infarction in Europe: description of the current situation in 30 countries. Eur Heart J 2010;31:943-57.

3. Velagaleti RS, Pencina MJ, Murabito JM, et al. Long-term trends in the incidence of heart failure after myocardial infarction. Circulation 2008;118:2057-62

4. Yellon DM, Hausenloy DJ. Myocardial reperfusion injury. $N$ Engl J Med 2007;357:1121-35.

5. Jones DA, Andiapen M, Van-Eijl TJ, et al. The safety and efficacy of intracoronary nitrite infusion during acute myocardial infarction (NITRITE-AMI): study protocol of a randomised controlled trial. BMJ Open 2013;3:e002813.

6. Lovell MJ, Yasin M, Lee $\mathrm{KL}$, et al. Bone marrow mononuclear cells reduce myocardial reperfusion injury by activating the PI3K/Akt survival pathway. Atherosclerosis 2010;213:67-76.

7. Chien KR, Olson EN. Converging pathways and principles in heart development and disease: CV@CSH. Cell 2002;110:153-62.

8. Liao R, Pfister O, Jain M, et al. The bone marrow-cardiac axis of myocardial regeneration. Prog Cardiovasc Dis 2007;50:18-30.

9. Blum B, Benvenisty N. The tumorigenicity of human embryonic stem cells. Adv Cancer Res 2008;100:133-58.

10. Clifford DM, Fisher SA, Brunskill SJ, et al. Stem cell treatment for acute myocardial infarction. Cochrane Database Syst Rev 2012;2:CD006536.

11. Orlic D, Kajstura J, Chimenti S, et al Mobilized bone marrow cells repair the infarcted heart, improving function and survival. Proc Natl Acad Sci 2001;98:10344-9.

12. Oh $\mathrm{H}$, Bradfute SB, Gallardo TD, et al. Cardiac progenitor cells from adult myocardium: homing, differentiation, and fusion after infarction Proc Natl Acad Sci USA 2003;100:12313-18.

13. Orlic D, Kajstura J, Chimenti S, et al. Bone marrow cells regenerate infarcted myocardium. Nature 2001;410:701-5.

14. Kamihata $\mathrm{H}$, Matsubara $\mathrm{H}$, Nishiue $\mathrm{T}$, et al. Implantation of bone marrow mononuclear cells into ischemic myocardium enhances collateral perfusion and regional function via side supply of angioblasts, angiogenic ligands, and cytokines. Circulation 2001;104:1046-52

15. Kobayashi T, Hamano K, Li T-S, et al. Enhancement of angiogenesis by the implantation of self bone marrow cells in a rat ischemic heart model. J Surg Res 2000;89:189-95

16. Nishida M, Li T-S, Hirata K, et al. Improvement of cardiac function by bone marrow cell implantation in a rat hypoperfusion heart model. Ann Thorac Surg 2003;75:768-73; discussion 773-4.

17. Richardson JD, Bertaso AG, Psaltis PJ, et al. Impact of timing and dose of mesenchymal stromal cell therapy in a preclinical model of acute myocardial infarction. J Cardiac Fail 2013;19:342-53.

18. Assmus B, Schächinger V, Teupe C, et al. Transplantation of progenitor cells and regeneration enhancement in acute myocardial infarction (TOPCARE-AMI). Circulation 2002;

106:3009-17.

19. Wollert KC, Meyer GP, Lotz J, et al. Intracoronary autologous bone-marrow cell transfer after myocardial infarction: the BOOST randomised controlled clinical trial. Lancet 2004;364:141-8.

20. Schachinger V, Erbs S, Elsässer A, et al. Intracoronary bone marrow-derived progenitor cells in acute myocardial infarction. N Engl J Med 2006;355:1210-21.

21. Janssens S, Dubois C, Bogaert J, et al. Autologous bone marrow-derived stem-cell transfer in patients with ST-segment elevation myocardial infarction: double-blind, randomised controlled trial. Lancet 2006;367:113-21.

22. Lunde K, Solheim S, Aakhus S, et al. Intracoronary injection of mononuclear bone marrow cells in acute myocardial infarction N Engl J Med 2006;355:1199-209.

23. Hirsch A, Nijveldt $R$, van der Vleuten PA, et al. Intracoronary infusion of mononuclear cells from bone marrow or peripheral blood compared with standard therapy in patients after acute myocardial infarction treated by primary percutaneous coronary intervention: results of the randomized controlled HEBE trial. Eur Heart $J$ 2011;32:1736-47.

24. Traverse JH, Henry TD, Ellis SG, et al. Effect of intracoronary delivery of autologous bone marrow mononuclear cells 2 to 3 weeks following acute myocardial infarction on left ventricular function: the LateTIME randomized trial. JAMA 2011;306:2110-19.

25. Traverse JH, Henry TD, Pepine CJ, et al. Effect of the use and timing of bone marrow mononuclear cell delivery on left ventricular function after acute myocardial infarction: the time randomized trial. JAMA 2012;308:2380-9.

26. Schachinger V, Assmus B, Britten MB, et al. Transplantation of progenitor cells and regeneration enhancement in acute myocardial infarction: final one-year results of the TOPCARE-AMI Trial. J Am Coll Cardiol 2004;44:1690-9.

27. Flett AS, Hasleton J, Cook C, et al. Evaluation of techniques for the quantification of myocardial scar of differing etiology using cardiac magnetic resonance. JACC Cardiovasc Imaging 2011; 4:150-6.

28. Petronio AS, Rovai D, Musumeci G, et al. Effects of abciximab on microvascular integrity and left ventricular functional recovery in patients with acute infarction treated by primary coronary angioplasty. Eur Heart $J$ 2003;24:67-76.

29. Surder D, Schwitter J, Moccetti T, et al. Cell-based therapy for myocardial repair in patients with acute myocardial infarction: rationale and study design of the SWiss multicenter Intracoronary Stem cells Study in Acute Myocardial Infarction (SWISS-AMI). Am Heart J 2010;160:58-64. 